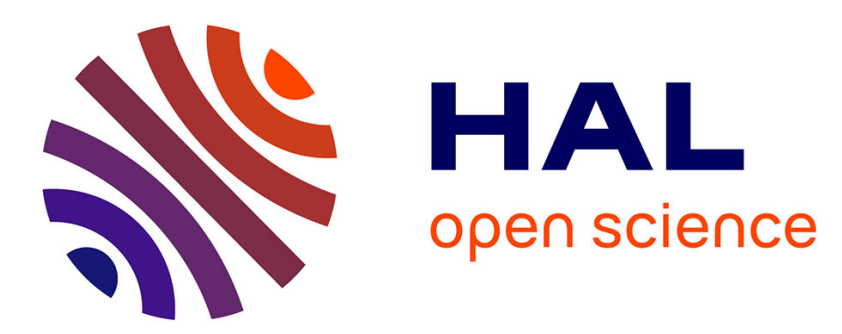

\title{
Mightability maps: A perceptual level decisional framework for co-operative and competitive human-robot interaction
}

Amit Kumar Pandey, Rachid Alami

\section{- To cite this version:}

Amit Kumar Pandey, Rachid Alami. Mightability maps: A perceptual level decisional framework for co-operative and competitive human-robot interaction. IEEE/RSJ International Conference on Intelligent Robots and Systems (IROS), Oct 2010, Taipei, Taiwan. hal-01977478

HAL Id: hal-01977478

https://hal.laas.fr/hal-01977478

Submitted on 10 Jan 2019

HAL is a multi-disciplinary open access archive for the deposit and dissemination of scientific research documents, whether they are published or not. The documents may come from teaching and research institutions in France or abroad, or from public or private research centers.
L'archive ouverte pluridisciplinaire HAL, est destinée au dépôt et à la diffusion de documents scientifiques de niveau recherche, publiés ou non, émanant des établissements d'enseignement et de recherche français ou étrangers, des laboratoires publics ou privés. 


\title{
Mightability Maps: A Perceptual Level Decisional Framework for Co-operative and Competitive Human-Robot Interaction
}

\author{
Amit Kumar Pandey and Rachid Alami
}

\begin{abstract}
Interestingly Humans are able to maintain a rough estimates of visibility, reachability and other capabilities about not only themselves but of the person they are interacting with. Studies in neuroscience and psychology suggests that from the age of 12-15 months children start to understand the occlusion of others' line-of-sight and from the age of 3 years they start to develop the ability, termed as perceived reachability for self and for others'. As such capabilities evolve in the children, they start showing intuitive and proactive behavior by perceiving various abilities of the human partner.

Inspired from such studies, which suggest that visuo-spatial perception plays an important role in human-human interaction, we propose to equip our robot with the capabilities to maintain various types of reachabilities and visibilities of itself and of the human partner in the shared workspace. Since these analyses will be basically perceived by performing a virtual action onto the agent and roughly estimating what that agent might be able to 'see' and 'reach' in 3D space, we term these representations as Mightability Maps.

By applying various set theory operations on weighted Mightability maps, robot could perceive a set of possible candidate solutions in real time for various tasks. We show its application in two completely different behaviors of robot: Cooperative and competitive behaviors. In the first case robot decides for two tasks: where to show an object to the human and where to put an object on the table for the Human. In the second case robot decides where to hide an object from human. These maps are quick to compute and could help in developing higher-level decisional capabilities in Robot.
\end{abstract}

\section{INTRODUCTION}

$\mathrm{W}$ E, the Humans, have the capability to perceive (imagine) various abilities of ourselves as well as of others in various situations. Suppose that Jack and Jill are sitting on the two sides of a table, cluttered with different objects, occluding their field of vision, as shown in fig. 1 . Suddenly Jack asks to Jill "please put the bottle near me". Jill picks and puts the bottle at an 'appropriate' place on the table where Jack could not only 'easily' see the bottle but also could 'easily' reach to the bottle. Now it's time for the Jill to counter-command: "show me the cup", which was actually occluded for her by the big object. Jack picks and holds the cup in the way Jill can 'easily' see it. Interestingly, neither Jill nor Jack took any pain in performing their tasks. The interesting fact is, however Jack was aware that if he will stand up and lean forward, he might be able to reach the bottle, but he was also aware that not only Jill could 'easily' reach the bottle without such efforts, but also she shares an

Authors are with CNRS; LAAS; 7 avenue du Colonel Roche, F-31077 Toulouse, France. Université de Toulouse; UPS, INSA, INP, ISAE; LAAS; F-31077 Toulouse, France. Email: \{akpandey,rachid.alami\}@laas.fr 'easily' reachable space with him. Similarly Jill was also aware that if she would stand up and move she might be able to see the cup, but she also chose the 'easy' way to see the cup, by perceiving Jack's capability. This suggests that they have some perception about the places, which could be reachable and visible by both, even before performing the actual task. For measuring such capabilities and assigning the level of 'ease' they basically associated and executed virtual actions (stand up, bend, move etc.) onto the agents and then perceived different abilities.

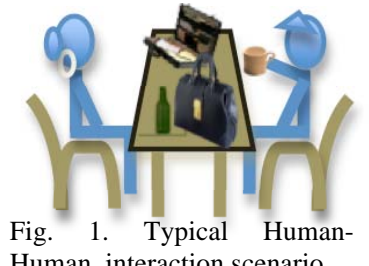

The above discussion points out following 3 important issues:

(i). Maintaining various types of abilities (reachability, visibility, ability to grasp an object etc.) of self and of others.

(ii). Assigning macro level 'ease' or 'comfort' to such abilities (reachable without leaning forward, visible without turning etc.) and finalizing mutual comfort level for performing a task, depending upon the current state, task as well as the role of the person (slave, friend, boss etc.).

(iii). Deciding micro level comfort (reachable without leaning but also near hand, visible without turning but also near the axis of field of view of person etc.)

This paper will mainly focus on first point, i.e. maintaining various types of visibility and reachability, which our robot will perceive by performing different types of virtual actions on itself and on the human partner. The second and third issues need to be addressed separately as they also require a proper formalization and evaluation for finalizing the optimal comfort, which depends upon the mental and physical states of the agent as well as the task and various other factors. So, for the experimental demonstration of our proposed solution for the issue (i), which in fact could help in developing the higher-level decisional capabilities in the robot, we will just assume the robot and human are co-operating each other and assign an intuitive level of macro and micro level comforts.

In this paper we will confine ourselves to two abilities: Visibility and Reachability. In psychology and neuroscience literatures the term visuo-spatial working memory has been explored, [5], and analyzed from the point of view of human reasoning [6]. Visuo-spatial perception is an important aspect of cognitive functioning because it is responsible for a wide range of activities of daily living. For instance, it involved in our ability to accurately reach for objects in our visual field and our ability to shift our gaze to different 
points in space. Studies, such as [1], suggest that from the age of 3 years, children are able to perceive which places are reachable by them and by others, as the sign of early development of allocentrism capability, i.e. spatial decentration and perspective taking. Moreover it is not sufficient to know which objects are visible for an agent, but also which space in $3 \mathrm{D}$ is visible to an agent. Imagine the case when we need to find place in $3 \mathrm{D}$ to show or hide something from other. At 12-15 months of age children also show evidence of an understanding of occlusion of others' line-of-sight [11], [12]; and an adult is seeing something that they are not when looking to locations behind them or behind barriers [13], [14]. Experiments in these studies show that infants in which these abilities to perceive visuo-spatial reasoning have not been developed yet, fail to help, cooperate or understand the intention of their adult partner.

Inspired from such evidences from neuroscience and psychology, in this paper we propose to equip our robot with such very basic but important capabilities of maintaining spatial and visual abilities of itself and of the human partner. This will facilitate the robot to behave more intuitively both in co-operative and competitive scenarios.

Representation of reachable and manipulable workspace has already received attention form various researchers. In [7], the kinematic reachability and directional structure for the robot arm has been generated. Although, it is an offline process with computation time of 12.4 hours, such representation has been shown useful in generation of reachable grasp [8]. In [9] an offline technique for mapping workspace to the configuration space for redundant manipulator has been presented, with the argument favoring to maintain multiple solutions. They chose a configuration according to the manipulability measure. In [10], a Monte Carlo based randomized sampling approach has been introduced to represent the reachable workspace for a standing humanoid robot. It stores the true or false information about the reachability of a cell by using the inverse kinematics. However none of these works focus on such analysis with different postural and environmental constraint as well as they don't calculate such abilities of the human partner, which is one of the important aspect for decision making in a Co-operative Human-Robot scenario.

Regarding the visual aspect of visuo-spatial reasoning, there have been works in the field of robotics mainly on perspective taking. In [19], a learning algorithm has been presented that takes into account the teacher's visual perspective. In [18], the perspective taking has been used to make more accurate action recognition. In [20] perspective taking has been shown to be useful in human-robot interaction and [17], we have shown the ability of the robot about geometric visual perspective taking about the human partner. But most of such works answer to the question: which object is visible not which spaces in the $3 \mathrm{D}$ are visible. In fact such object level visibility will be used in deciding upon the final point in $3 \mathrm{D}$ space for performing a specific task. Our proposed map of visibility in $3 \mathrm{D}$ space is complementary to such object level visibility analysis.

In psychology [21], [22], in Human-Computer Interaction [23] and in Robotics [24], [25], term affordance is used, which basically associates with object in the environment from the perspective of task and/or agent. In our knowledge there is no significant work has been published in analyzing various types of visibility and reachability from the robot as well from human point of view and combining them for various decision making. In this paper we propose the term Mightability Map as the representation of various perceived abilities of the agent in 3D, which is independent of manipulation task or object to a certain extent.

In the next section we will describe different abilities the robot will perceive and how it calculates them. Although associating a psychological or physical comfort level to these perceived abilities are not the scope of this paper, just for the sake of demonstration we will intuitively rank these perceived abilities. In section III, we will show their usefulness for performing the co-operative tasks of "putting the bottle" and "showing the bottle" to the Human as well as competitive task of "hiding the bottle" from human in a face to face Human-Robot Interaction scenario, by our Humanoid robot HRP2. Section IV will contain some discussion on other potential applications of our proposed Mightability Maps followed by the conclusion and future works.

\section{Mightability MaP : The PERCEIVED ABILITY}

\section{A. Mightability Map}

The main motivation behind the present work is to maintain a set of knowledge about different abilities, as humans do, which: (i). could be fast to compute, (ii). should not underestimate any ability, as not to miss any solution for a task if one exists, (iii). provide a relevant basis for semantic reasoning capability to robot, (iv). is independent to the nature of the task/human activity, which might not be known in advance and even help the robot to predict it. (v). facilitate robot to behave/communicate more intuitively in real time with the human partner.

Since, various abilities of the agent will be perceived by applying some virtual actions on it, hence it will inherit overestimation/uncertainty for the sake of not being underestimated. We term it as Mightability (for Might be Able, a rough estimation) Map (for representing in 3D grid).

Each Mightability map will answer to a particular question about the perceived ability, for example "if the human will lean forward, he/she might be able to reach these places", "if human will turn around he might be able to see these places". As will be shown, one of the immediate benefits of such Mightability maps is, it will greatly reduce the search space, hence the time for finding a point for performing a particular task, as well as maintain a set of candidate $3 \mathrm{D}$ points of possible solution.

\section{B. Perceived Reachability}

From the studies in [2], [3], [4] the general agreement is that, the prediction to reach a target with the index finger 
depends on the distance of the target relative to the length of the arm, which slightly overestimates actual arm length by about $10 \%$, but plays as a key component in actual movement planning. We will directly use their findings and use the length of the arm to estimate the reachability boundary for the given postural situation of the human and the robot. An agent (Human or Robot) can show reaching behavior to touch, to grasp, to push or hit an object or just to point something, or to take something from inside some container. Hence, having a perceived maximum extent of the agent's reachability even with some overestimation will be acceptable as the first level of estimating the ability. As will be shown in the section III, these perceived reachabilities could then be filtered by the nature of the task as well as more rigorous kinematics and dynamics constraints.

In the
studies of
human
movement and
behavioral
psychology,

[16], [17]

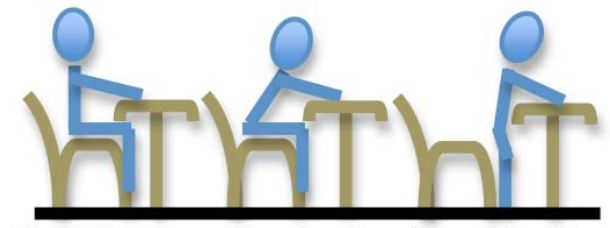

Fig. 2. Taxonomy of reach actions:(a) arm-shoulder different types of reach action of the human has been identified and analyzed. Ranging from a reach involving simple arm extension without any other body movement (arm-only reach), shoulder extension (arm-and-shoulder reach) or leaning forward (arm-and-torso reach) and stand in order to pick up the object (standing reach). Fig. 2 illustrates taxonomy of such reach actions. One of the interesting observations is that if human is sitting very near to the table, it will be able to lean less as compared to sitting away from the table, hence sometimes regions reachable in former case will be less as shown in fig 3(a) \& (b). While generating the Mightability map we will also take into account various postural (Sitting, standing) and 3D environmental (table, chair etc) constraints as well as collision checking.

We have
augmented
various other
reachability
analyses to the
set proposed in

[16]: reaching by turning around

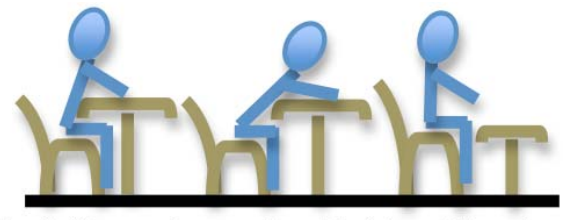

Fig. 3. Hunnan clusen to the table (a) cuuld lean less compared to sitting away from the table (b). Table is too low to be reached without leaning (c).

only and reaching by turning around and then leaning forward, for sitting as well as for standing. Furthermore, the robot distinguishes the places, only reachable by left hand or right hand or by both hands of the agent. And all these analyses are done for 3D space around the robot and human, not only for a table surface. In computing such reachability the joint limits of shoulder, neck and waist of HRP2 and of Human is taken care of. Values of such limits for an average human as well as for HRP2 have been taken from [26]. The robot computes such abilities in the following manner:

Robot is having the structure of the kinematics chain of itself and of the human. As shown in the fig. 4, information

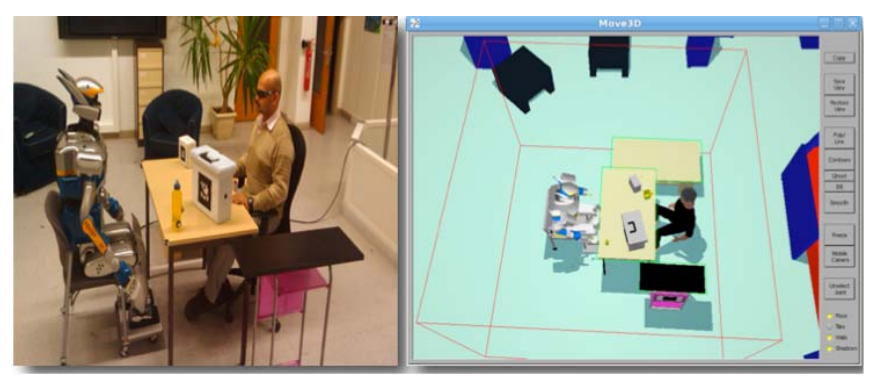

Fig. 4. (a) Experimental setup (b) 3D map of environment, which the robot maintains and uses for decision making and planning.

about the human position, orientation and the 3D structure of the environment, fig. 4(a), is continuously updated in our 3D platform, Move3D, fig. 4(b), as will be described in the section III-C. In our Move3D environment robot can check collisions among various parts of itself and human model as well as objects. Robot construct a 3D box around it (shown as red box fig. 4(b)), sufficient enough to enclose the human, tables and other objects defining the current workspace, (dimension of $3 m \times 3 m \times 2.5 m$ for current scenario) and divides it into grid cells, each cell having dimension of $5 \mathrm{~cm}$ $x 5 \mathrm{~cm} \times 5 \mathrm{~cm}$.

First of all robot calculated the armshoulder reach. For this robot stretches the hand of the $3 \mathrm{D}$ model of human by permissible limit of each shoulder's yaw and pitch

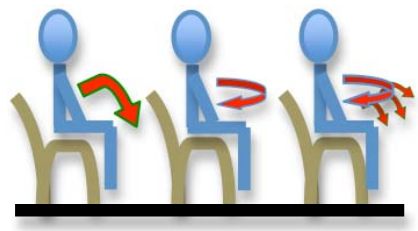

Fig. 5. Leaning, turning-around, turning-around-and-leaning torso. joints and computes which cells in the 3D grid are reachable by human just by using the length of the arm from the shoulder to finger. Then the robot virtually leans the human model by its torso incrementally until there is a collision with the table or any other object or the maximum limit of human waist pitch joint has been reached. Then from these new virtual positions of the human, robot again calculates the reachability in $3 \mathrm{D}$ as explained earlier. Then the robot turns the torso of the human model at its current position until collision or maximum limit of human waist yaw is reached, to calculate the reaching by turning-around. Similarly the reachability of turning-around-and-leaning is computed. Robot also calculates all these reachabilities by virtually making the human standing as well as for itself.

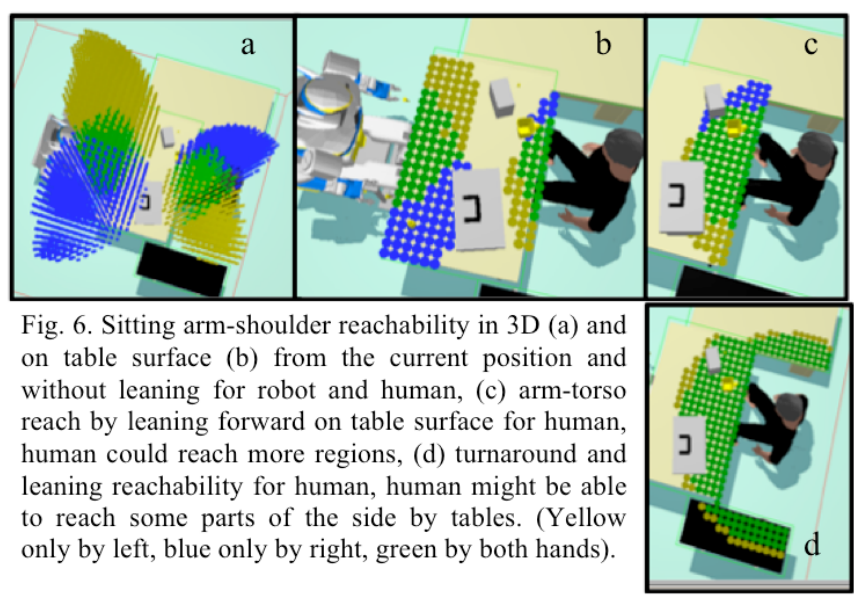


Fig. 5 illustrates few such reachabilities calculations.

Fig. 6(a) shows the arm-shoulder reachable cells in 3D for HRP2 and Human from their current position and orientation. Robot also distinguishes among the cells, which could be reached only by left hand (yellow), only by right hand (blue) and by both hands (green). Fig. 6(b) show similar cells but only belonging to table surface. It could be easily seen that there is no common reachable region on table if neither of them will lean forward. Fig. 6(c) shows reachability of human on table but with maximum possible leaning forward. Clearly the human might be able to reach more regions. The robot is able to also perceive that if human will turn and lean he will be able to reach some parts of the side by tables which are of different heights, as shown in fig. 6(d). Note that at this level all the cell which are reachable are estimated, irrespective of the fact, is there any object or it is free space, because as mentioned earlier, robot should be able to predict reachability for a range of tasks.

\section{Perceived Visibility}

For calculating the visibility, from the current position and yaw and pitch of the head, robot finds the plane perpendicular to the axis of field of view. Then

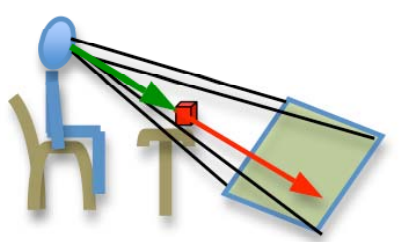

Fig. 7. Calculation of visibility that plane is uniformly sampled to the size of a cell of the 3D grid of Mightability map. Then as shown in fig. 7, a ray is traced from the eye/camera of the agent to each such sample on the plane. If an obstacle is found on the way, all the cells of the corresponding Mightability map till the obstacle (green arrow) is marked as visible and rest of the points on the ray till the plane (red arrow) is marked as invisible. The different types of virtual actions, which the robot performs on the agent to perceive various Mightability Maps are: only turn the head till the joint limit of neck's yaw and pitch, turn the torso as well as head until collision or till the limit of waist yaw, both for sitting and standing.

Red circles in fig. 8(a) shows the visibility of the human from his current position and head orientation for the cells of corresponding 3D Mightability Map, which are on the table

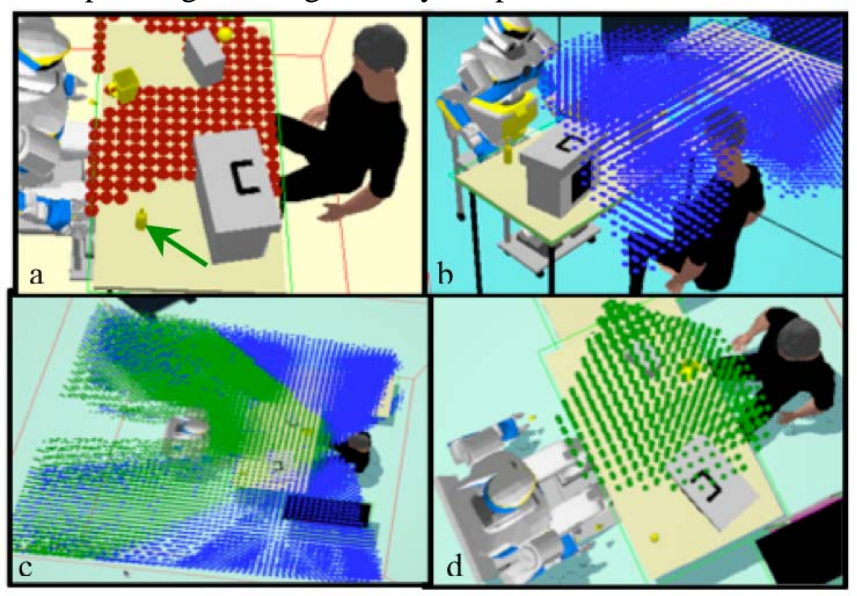

Fig. 8. Current visibility of human on table surface (a) and of HRP2 in 3D (b). (c) Current and turning the head around visibility of human without turning torso. (d) Common current visibility of Human and robot in 3D. plane. Note the invisible regions because of the objects. The blue cells in fig. 8(b) show the visibility in 3D for the HRP2 robot for its current orientation of head. Fig. 8(c) shows the current visibility (green cells) as well as the visibility when human will turn its head without turning the torso (blue cells) in 3D. Fig. 8(d) shows the current common visibility of HRP2 and Human in 3D. As the field of view of the HRP2 camera is different from the human's eye, the corresponding Mightability maps will not be symmetric.

\section{Perceived Mightability Maps and Computation Time}

Table I summarizes different types of perceived Mightability Maps for reachability and visibility computed by the robot for about itself as well as about the human. Table II shows time for calculating various Mightability maps. It also shows the time for one time process of creating and initializing cells of the 3D grid to discretize the workspace with various information like cells which are obstacle free, which contains obstacles, which are the part of the horizontal surfaces of different tables etc. Note that it took 1.6 seconds to create and initialize 3D grid consisting of $180000(60 \times 60 \times 50)$ cells, each of dimension $5 \mathrm{~cm} \times 5 \mathrm{~cm} x$ $5 \mathrm{~cm}$, hence 0.000009 seconds for a single cell. Assuming that most of the changes in the environment will affect a fraction of the $3 \mathrm{D}$ grid, like movement of objects on the table and/or change in position of some body parts of the agents, the Mightability Map set could be updated quickly. From table II, it can be seen for an average scenario as shown in fig. 2 it takes about 0.446 seconds to calculate all the Mightability maps for human and robot, once the 3D grid is initialized.

\begin{tabular}{|c|c|c|}
\hline \multicolumn{3}{|c|}{$\begin{array}{l}\text { TABLE I } \\
\text { RCEIVED MIGHTABILITY MAPS }\end{array}$} \\
\hline \begin{tabular}{lll|} 
Visual & 3D & Mightability \\
Maps: For & HRP2 and \\
Human & &
\end{tabular} & \multicolumn{2}{|c|}{$\begin{array}{l}\text { Spacial 3D Mightability Maps: For } \\
\text { HRP2 and Human (by only left hand, } \\
\text { by only right hand and by both hands) }\end{array}$} \\
\hline $\begin{array}{l}\text { From Current } \\
\text { orientation, (C) }\end{array}$ & \multicolumn{2}{|c|}{$\begin{array}{l}\text { Arm-shoulder reach from } \text { Current } \\
\text { position, (C) }\end{array}$} \\
\hline $\begin{array}{l}\text { Virtually Turning the head } \\
\text { around until collision or } \\
\text { Neck's yaw joint limit is } \\
\text { reached, (T N) }\end{array}$ & \multicolumn{2}{|c|}{$\begin{array}{l}\text { Arm-torso reach by virtually Leaning } \\
\text { torso until collision detected or Waist's } \\
\text { pitch joint limit is reached, (L_W) }\end{array}$} \\
\hline \multirow{2}{*}{$\begin{array}{l}\text { Virtually Turning and } \\
\text { Leaning the torso until } \\
\text { collision or Waist's joint } \\
\text { limits are reached and then } \\
\text { turn Neck until collision or } \\
\text { neck's yaw joint limit is } \\
\text { reached, (T_W_N) }\end{array}$} & \multicolumn{2}{|c|}{$\begin{array}{l}\text { Arm-shoulder reach by virtually Turning } \\
\text { around until collision detected or Waist's } \\
\text { yaw joint limits are reached, (T_W) }\end{array}$} \\
\hline & \multicolumn{2}{|c|}{$\begin{array}{l}\text { Arm-torso reach by virtually Turning } \\
\text { around and Leaning torso until collision } \\
\text { detected or Waist's yaw and pitch joint } \\
\text { limits are reached, (T_L_W) }\end{array}$} \\
\hline 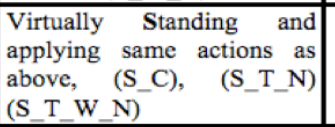 & \multicolumn{2}{|c|}{$\begin{array}{l}\text { Virtually Standing and applying same } \\
\text { actions as above, (S_C), (S_L_W), } \\
(\mathrm{S} \text { T_W), (S_T_L_W) (this row is not } \\
\text { applicable for HRP2 if sitting) }\end{array}$} \\
\hline \multicolumn{3}{|c|}{$\begin{array}{l}\text { TABLE II } \\
\text { MIGHTABILITY MAPS IN SECONDS (S) }\end{array}$} \\
\hline \multicolumn{2}{|c|}{ 3D grid creation and initialization (one time process) } & 1.6 \\
\hline \multicolumn{2}{|c|}{$\begin{array}{l}\text { 3D visual Mightability Maps for human } \\
\left(\mathrm{C}+\mathrm{T} \_\mathrm{N}+\mathrm{T} \_\mathrm{W} \_\mathrm{N}+\mathrm{S} \_\mathrm{C}+\mathrm{S} \_\mathrm{T} \_\mathrm{N}+\mathrm{S} \_\mathrm{T} \_\mathrm{W} \_\mathrm{N}\right)\end{array}$} & 0.146 \\
\hline \multicolumn{2}{|c|}{ 3D visual Mightability Maps for Robot $\left(\mathrm{C}+\mathrm{T} \_\mathrm{N}+\mathrm{T} \_\mathrm{W} \_\mathrm{N}\right)$} & 0.089 \\
\hline \multicolumn{2}{|c|}{$\begin{array}{l}\text { 3D spatial Mightability Maps for human } \\
(\mathrm{C}+\mathrm{L} \quad \mathrm{W}+\mathrm{T} \quad \mathrm{W}+\mathrm{T} \quad \mathrm{L} \quad \mathrm{W}+\mathrm{S} \quad \mathrm{C}+\mathrm{S} \quad \mathrm{L} \quad \mathrm{W}+\mathrm{S} \quad \mathrm{T} \text { W }+\mathrm{S} \quad \mathrm{T} \text { L W) }\end{array}$} & 0.128 \\
\hline \multicolumn{2}{|c|}{$\begin{array}{l}\text { 3D spatial Mightability Maps for Robot } \\
\text { (C+L_W }+ \text { T_W }+ \text { T_L_W })\end{array}$} & 0.083 \\
\hline
\end{tabular}




\section{APPLICATION, EXPERIMENTS AND RESULTS}

\section{A. Representing Information from Mightability Maps}

We use following convention to represent these Mightability Maps: The Mightability Map computed for Human and for Robot will have prefix ' $\mathrm{MH}$ ' and ' $\mathrm{MR}$ ' respectively, which will be followed by ' $V$ ' or ' $S$ ' for visual or special aspect. Then the acronyms of map type as given in the table I will be added as suffix. For example, the spatial Mightability Map for perceiving the human reach by maximum possible turning around and leaning by waist will be represented by MHST_L_W. So, the set of all the Mightability Maps for human is represented as:

$M H=\{m: m \in M H V \vee m \in M H S\}$, where, $M H V=\left\{M H V{ }_{-} C, M H V{ }_{-} T{ }_{-} N, M H V_{-} T_{-} W_{-} N\right.$, $\left.M H V_{-} S_{-} C, M H V_{-} S_{-} T_{-} \bar{N}, M H V_{-} S_{-} T_{-}^{-} W_{-} N\right\}$, and $M H S=\left\{M H S_{-} C, M H S_{-} L_{-} W, M H S_{-} T_{-} W, M H S_{-} T_{-} L_{-} W\right.$, $\left.M H S_{-} S_{-} C, M H S_{-} S_{-} L_{-} W, M H S_{-} S_{-} T_{-} W, M H S_{-} S_{-} T_{-} L_{-} W\right\}$

The set of all the Mightability Maps for robot, $M R$, is represented in similar manner.

Since, as mentioned earlier, for exhibiting various cooperative as well as competitive behaviors, the Mightability Maps not only encode information about what an agent might be able to do, but also what he/it might not be able to do. We assume an operator ' $\operatorname{val}((x, y, z), M)$ ', which returns the value 1 (might be able) or 0 (might not be able) for a particular cell $(\mathrm{x}, \mathrm{y}, \mathrm{z})$ of a particular Mightability map $\mathrm{M}$. Hence, for example the set of all cells, to which human might be able to arm-shoulder-reach from his current position, will be denoted as:

MHS_C_True $=\left\{(x, y, z):(x, y, z) \in M H S \_C \wedge v a l\left((x, y, z), M H S \_C\right)=1\right\}$

\section{B. Using Mightability maps}

Fig. 9 illustrates, how such Mightability map could be potentially used to get a fast and optimal solution for particular task. We will describe the steps in fig. 9, in the context of a particular task of putting a bottle near the human. As shown fig. 8(a), the yellow bottle indicated by arrow is presently not visible to the human, and let us assume that based on current context the robot has predicted that human might need that bottle. So, the robot's task will be to 'make' the bottle visible and reachable to the human.

By analyzing various Mightability Maps, which are easy and quick to compute for both the agents, robot will have a rough idea about reachability and visibility of the bottle, from the perspective of both agents. Robot will be able to

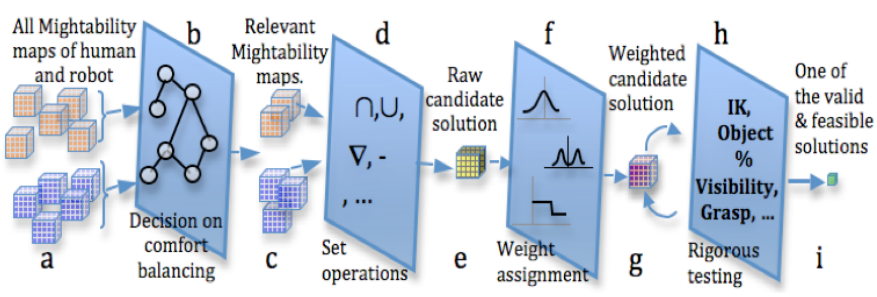

Fig. 9. (a) Initial Mightability Maps, (b) Decision making, (c) Relevan Mightability maps, (d) Set operations (e) Raw candidate solution set, (f) Weight assignment to get a set of weighted candidate points (g), for a particular task. (h) Applying rigorous and expensive tests on weighted candidate points to get the final optimal solution (i), of highest weight. know that, if it will ask human to stand up and lean, human will be able to see as well as reach the bottle. Alternatively, robot will be also aware that it could also reach the bottle and make the bottle visible and reachable to human by putting it 'somewhere' on the table near the human. As mentioned earlier deciding upon the mutual balanced comfort level for achieving a particular goal requires separate investigation. Fig. 9(b) shows such decision making step on macro level comfort. We will simplify this step by assuming that robot is cooperating the human as a partner but also aware of its limitations, such as it can not stand up by itself from the chair and can not turn the torso significantly as it will collide with the chair. Hence it has decided to achieve the goal:

(i) by taking the bottle by the nearest hand reachable to it, and putting it at a place on any table's surface, which is

(ii) directly arm-shoulder reachable by robot from its current position, needs MRS_C,

(iii) AND visible by robot without any need for turning the torso, needs MRV_C and MRV_T_N,

(iv) AND either arm-shoulder reachable by either hand of the human from his current position, needs MHS_C,

(v) or reachable by either hand of the human by only leaning forward, needs MHS_L_W,

(vi) AND visible by human from his current position and orientation, without any need for him to turn around, i.e. in his current field of view, needs MHV_C.

With such criteria, robot will get a subset of relevant Mightability maps as shown in the fig. 9(c). In our case it will be MRS_C, MRV_C, MRV_T_N, MHS_C, MHS_L_W and MHV_C. Now the next step is to apply various set operations, fig. 9(d), on these relevant Mightability maps to get the set of candidate cells. For our case the set of candidate cells will be given by:

Raw_Candidate_Cells $=\left(M R S_{-} C_{-}\right.$Ture $\left(M R V_{-} C_{-} T r u e \bigcup M R V{ }_{-} T_{-}\right.$

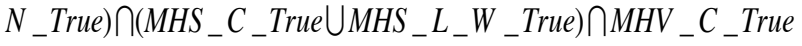

where, MRS_C_True is set of cells of Mightability map $M R S \_C$, whose values are 1 , which is denoted as:

$M R S_{-} C_{-}$True $=\left\{(x, y, z):(x, y, z) \in M R S_{\_} C \wedge \operatorname{val}\left((x, y, z), M R S \_C\right)=1\right)$ and similarly for others.

Since, the task is to put the bottle on any of the table surface, the final set of candidate cells are further reduced: Raw_Candidate_cells_to_Put $=\{(x, y, z):(x, y, z) \in$ Raw

_Candidate_Cells $\wedge(x, y, z) \in \bigcup_{i=1}^{n}$ surface_of_table $\left._{i}\right\}$

where $n$ is the total number of tables inside the bounding box of the 3D grid and surface_of_table ${ }_{i}$ represents the horizontal supporting plane of table $\mathrm{i}$ in the environment, information about which is already encoded in the 3D grid during the process of creation and initialization.

Hence robot quickly gets a set of candidate cells, fig. 9(e), within which the feasible solutions will lie if exist. Also as table III shows, the search space for the rigorous testing for finding the final solution has been significantly reduced.

Now, after having a candidate set of cells, one could directly jump to the testing step by assuming equal weight for each cell, or could further assign micro level weights, fig. 
9(f), within the candidate search space, to get a weighted candidate cells, fig. 9(g). As discussed in section I, assigning such weights depend on various criteria and need investigation, we will assign two intuitive measures of preference for the candidate cells to put the bottle. (i) Assign higher weights for the points which are along the front axis of the human torso, by $2 \mathrm{~d}$ Gaussian distribution centered at a distance of $0.35 \mathrm{~m}$ from human along this axis, to avoid putting very close to the human (ii) Then add higher weights for the points which are near to the current position of the bottle, by Gaussian distribution centered at bottle's position.

After having a set of weighted candidate points, robot performs various feasibility tests on each point in the order of highest to lowest weights. For the current scenario the robot performs following tests: (i) Is there any feasible configuration for the robot to grasp the bottle, which also satisfies the task constraints? (ii) Will the bottle be significantly/clearly visible to the human if put at that point? (iii) Will the robot be able to put the bottle at that point by avoiding collision as well as respecting its kinematic and dynamic constraints? For the current implementation we simplify (i) by assuming that any cylindrical grasp will be acceptable. For (ii) we use our perspective taking system [17] to calculate the $\%$ visibility of the object, if placed at a particular position and orientation. For (iii), we use our Generalized Inverse Kinematics library and smooth and collision free planning modules to plan whole body motion of HRP2.

In the current implementation, we use the first candidateweighted point, which passes through all the abovementioned rigorous and expensive tests, as the final point for performing the current task. As all these tests when combined together, becomes expensive for a single cell and if this has to be done for all the cells in the workspace, it would have been very expensive. In that case either the robot has to plan and find solution offline or use some randomized search algorithm, which would provide a solution not necessarily the optimal one. Hence, Mightability Maps as a decisive and reasoning tool on the candidate search space greatly reduces the search space for a particular task and the robot could quickly converge to the most preferred solution, which is $0.34 \mathrm{~s}$ for current example, as shown in table III.

\section{Experimental Setup and Testing}

We have tested our approach both in simulation and on real humanoid robot HRP2. Experimental setup is already shown in Fig. 4(a), which consists of 3 tables of different heights. The human and the robot sit in different configurations around the tables. The 3D map of the static environment is known to the robot, which includes heavy furniture and walls. The acquisition of the dynamic objects such as the big and small boxes and the cup is done by simple stereo-vision based system through robot's camera, which identifies and locates objects based on the visual tags. For tracking the human and the bottle we use motion capture system installed in the environment, which tracks the makers on bottle and on the frame of eyeglass human is wearing. To avoid the impression of bird's eye view, we pass the information about markers from motion capture system only if the markers are in the field of view of the robot. The positions and the orientations of the corresponding 3D models of the objects and the human are updated in Move3D environment in real time, which is the integrated 3D representation and planning platform developed at LAASCNRS, as shown in the fig. 4(b). Human gaze is simplified to the human head orientation and the length of human arm is assumed to be fixed in current implementation, however this could be calculated approximately by robot based on the detected height of the human. The robot first scans the environment by looking at different parts of it and updates the information about the objects on the table in its 3D representation of Environment. As well as perceives and maintains various Mightability Maps mentioned in table I. At some point of time, we assume that the robot predicts the task to put the bottle on an appropriate place, so that human could take it if required. Then as explained in previous subsections robot decides upon the final point to put the bottle. Fig. 10 shows the sequence of performing the task from initial to grasping to putting the bottle. Note that in fig. $10(d)$, which shows small set of weighted candidate points by green circles, the final solution to put the bottle is the cell which is away from the box, this is because of the criteria of significant $\%$ visibility of the bottle from the human perspective.

Fig. 11 shows a different scenario in which human is sitting on the right side of the robot and the smaller black table is placed in front of it. As shown in fig. 11(b) the feasible and most preferred solution lies on the table other than the table on which the bottle was initially placed and hidden from the human. As the Mightability Maps contain the visuo-spatial information of the $3 \mathrm{D}$ workspace volume, robot was able to easily associate the surface of the another table with the candidate solution and found the feasible solution to put the bottle so that human could easily see and take it whenever required.

To show the generic applicability of the Mightability Maps we have performed another experiment to show the bottle to the human by placing it appropriately in 3D space. A similar formulation for this task has been done but by

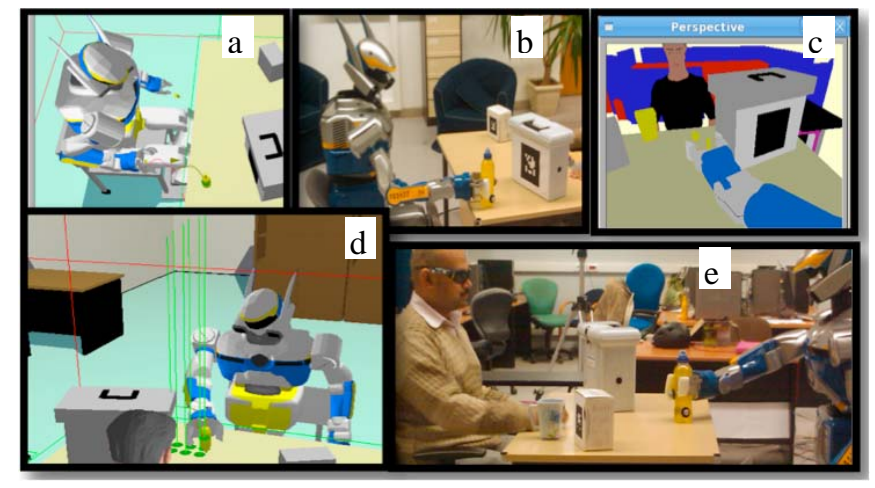

Fig. 10.(a)(b) Grasping the bottle by planning collision free path (c) Robots perspective at the time of putting the bottle. (d) Green circles show Candidate points to put the bottle, length of green line shows the weight of that point, (e) final place where the robot is putting the bottle for human. 


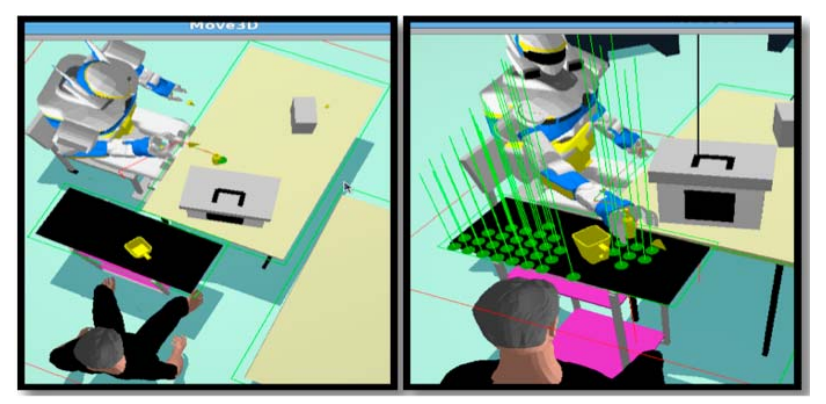

Fig.11. Task is to put the bottle for human to take (a) Initial position of the bottle (b) Candidate cells (green circles), their relative weights (length of green lines) and the final place to put.

relaxing few criteria. It is no loner necessary to be reached by human as well as the solution need not to be lying on the table surface. Hence the set of candidate cells will be : Raw_Candidate_Cells_to_Show $=\left(M R S_{-} C_{-} T u r e \bigcap\left(M R V C_{-}\right.\right.$True $U$

$\left.M R V_{-} T_{-} N_{-} T r u e\right) \bigcap M H V_{-} C_{-}$True)

And similar weights will be assigned to these points with few modifications. Now the yaw and pitch of the human head to see the point will be used as parameters for distributing the Gaussian weights, with the mean centered at the $0.5 \mathrm{~m}$ in front of human at the horizontal plane of human's neck (here we don't provide the details of our choice for weights for the sake of not deviating from current scope of this paper). Fig. 12(a) shows the initial and the set of weighted candidate points and fig. 12(b) shows final position at which the robot is showing the bottle to human.

As another attempt to show the competence of the Mightability Maps, we have designed a task in which the robot has to exhibit the competitive behavior of hiding the bottle somewhere on the table, so that the human could neither see it nor reach it from the sitting position, but it should be visible and reachable to the human if he stands up. The candidate points for this task will be given as :

Raw_Candidate_Cells_to_Hide $=\left((x, y, z):(x, y, z) \in\left(M R S_{-} C \_\right.\right.$Ture $\bigcap$

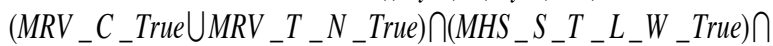

(MHV_S $\left.S_{-} T_{-} W_{-} N_{-} T r u e\right)-\left(\left(M H V_{-} C_{-} T r u e \bigcup M H V_{-} T_{-} N_{-}\right.\right.$True

$\left.M H V{ }_{-} T_{-}{ }_{-} N_{-} T r u e\right) \bigcup\left(M H S_{-} C_{-} T r u e \bigcup M H S_{-} L_{-} W_{-} T r u e \bigcup M H S\right.$ _

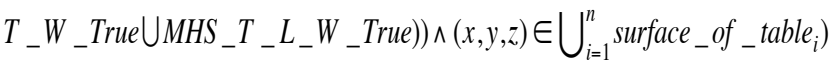

Note that the set of visible and reachable points from the sitting position of the human have been subtracted to get the set of points which are exclusively visible and reachable when the human stands up. Fig. 13 shows the sequence of hiding the bottle by putting it at the feasible place selected from the candidate places shown as green circles. Note that the clusters of candidate points on the left side of the robot in fig. 13(b) have been iteratively disqualified because of criteria of visibility from human perspective as well as

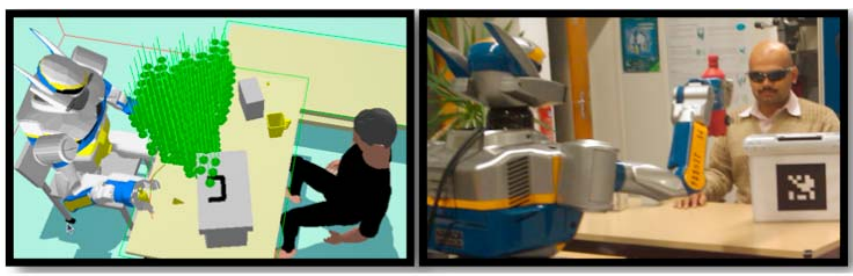

Fig. 12:(a) Candidate points in 3D to show the bottle, which was actually hidden from human (b) Robot showing the bottle at the feasible place.

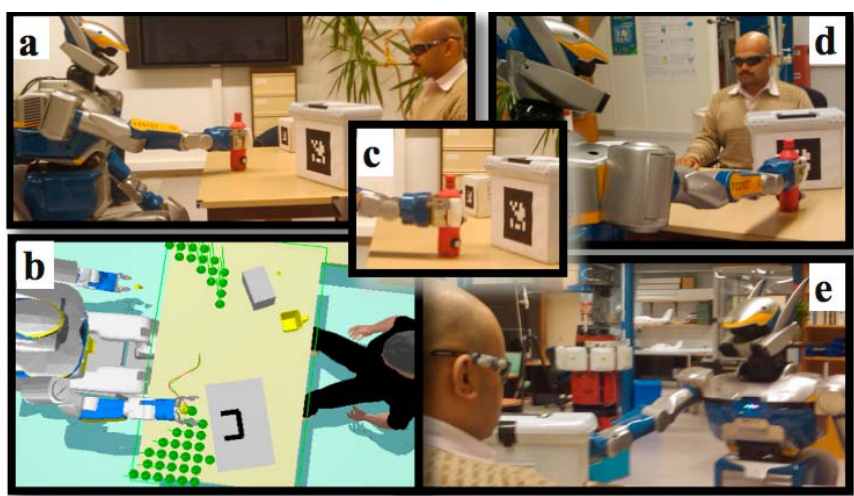

Fig. 13. Hiding the bottle from the human. (a) Initial position of bottle, (b) \& (c) hiding the bottle, (d) candidate places to hide the bottle, (e) $\&$ (f) bottle hidden from human field of view. Length of green lines in (f) shows the relative weights of the candidate places.

\begin{tabular}{|l|c|c|}
\hline \multicolumn{3}{|c|}{ TABLE III } \\
Task & $\begin{array}{c}\text { Significantly } \\
\text { Reduced Search } \\
\text { Space (number of } \\
\text { candidate cells) }\end{array}$ & $\begin{array}{c}\text { Time to get the first } \\
\text { feasible solution of } \\
\text { Maximum Weight } \\
\text { (in s) }\end{array}$ \\
\hline Initial Total Number of Cells = 144000 & 0.34 \\
\hline Put the bottle, fig. 10 & 8 & 0.52 \\
\hline Put the bottle, fig. 11 & 33 & 0.33 \\
\hline Show the bottle, fig 12 & 414 & 0.28 \\
\hline Hide the bottle, fig 13 & 42 & \begin{tabular}{c} 
(13) \\
\hline
\end{tabular}
\end{tabular}

collision free whole body motion testing of the robot.

Table III shows the candidate number of cell at the step (e) of fig 9, which is significantly less than the total number of cells. Also the time to obtain the highest weighted feasible solution, which in fact is the time of all the steps starting from fig. 9(a) till 9(i), including the iterative loop between 9(g) and 9(h), is reasonably acceptable for Human Robot Interacton.

The related videos could be found at http://homepages.laas.fr/akpandey/HRI_Videos.htm

\section{Discussion And Potential APPliCATIONS}

In fig. 9, we have not shown feedback and re-evaluation loops among various steps for the sake of presenting the basic framework within the scope of this paper. Also there could be loops between some external planner or decisionmaking system such as symbolic planner [31] or supervisor, which could iterate/query on the presented basic framework to find an alternative solution or maintain a ranked set of multiple solutions.

As these Mightability maps are fast to compute, they could also be stored as facts in chronicle based decision systems for higher-level reasoning. For example if the robot has information about the number of agents and their different abilities with time stamp and if there occurred some change in the environment about which the robot was oblivious, then in later point of time robot could infer which agent was capable of making that change. So, we are in the process of representing Mightability maps as facts in our control architecture [27]. These maps could also be used for 
development of shared plan, as well as bridging the gap between the symbolic and geometric planner [28], [29]. We are also in the process of integrating with our symbolic shared planner [30]. Furthermore the robot could use these maps for monitoring, understanding and predicting the probable actions and next step of the human partner. As studies in neuroscience and psychology suggests about children, all these will lead towards proactive robot.

\section{CONCLUSION AND FUTURE WORK}

We have introduced the concept of Mightability Maps, which immediately serves as a tool for visuo-spatial reasoning and decision making for various tasks in the human-robot interaction. We have shown that as these maps are independent of the type of manipulation task and are overestimation of different abilities, the feasible solution for a particular task will lie within these maps while greatly reducing the search space for rigorous and expensive feasibility checks. This makes the system to work without need of any offline preprocessing. We have also shown that various task oriented weights and set operations could be performed on these maps to get a set of candidate solutions.

Our proposed Mighatibility Map could be used not only for decision making for the cases requiring common abilities of the agents but also to solve the problems involving what another agent could not do. We have shown its use in cooperative as well as competitive scenarios. Another advantage of our presented framework is since it is not a randomized approach; hence instead of getting a random solution, it gives the feasible solution in the order of preference, which is provided to the system as weights.

Apart from the potential applications and ongoing works discussed in section IV, an immediate future work is to extend the building of such maps for more than two agents to facilitate the reasoning on multi-agent task allocation. It would also be interesting to develop methods, which provides the robot with the capability to decide autonomously about the types of Mightability Maps, which need to be updated periodically and which need to be calculated specifically for a particular task.

\section{REFERENCES}

[1] P. Rochat, "Perceived reachability for self and for others by 3- to 5year-old children and adults," Journal of Experimental Child Psychology, vol. 59, Issue 2, April 1995, pp. 317-333.

[2] C. Carello, A. Grosofsky, F. D. Reichel, H. Y. Solomon and M. T. Turvey, "Visually Perceiving What is Reachable," Ecological Psychology, Volume 1, Issue 1, March 1989, pp. 27-54.

[3] R. J. Bootsma, F. C. Bakker, F. J. Van Snippenberg, and C. W. Tdlohreg, "The Effects of Anxiety on Perceiving the Reachability of Passing Objects, "Ecological Psychology, Vol. 4(1), 1992, pp. 1-16.

[4] P. Rochat, and M. Wraga, "An account of the systematic error in judging what is reachable," Journal of Experimental Psychology: Human Perception and Performance, vol.23(1),Feb 1997, pp.199-212.

[5] T. Vecchi, M. L. Monticellai and C. Cornoldi, "Visuo-spatial working memory: Structures and variables affecting a capacity measure," Neuropsychologia, Vol. 33(11), Nov 1995, pp. 1549-1564.

[6] B. Tversky, (2005), "Visuospatial reasoning," In K. Holyoak and R. Morrison (Eds.), The Cambridge Handbook of Thinking and Reasoning,(pp. 209-240),Cambridge,MA:Cambridge University Press.
[7] F. Zacharias, Ch.Borst and G. Hirzinger, "Capturing robot workspace structure: representing robot capabilities," in Proceedings of the IEEE/RSJ IROS, pp. 3229-3236, San Diego, USA, October, 2007.

[8] F. Zacharias, Ch.Borst and G. Hirzinger, "Online Generation of Reachable Grasps for Dexterous Manipulation Using a Representation of the Reachable Workspace," in Proceedings of the International Conference on Advanced Robotics, Germany, 2009.

[9] L. Guilamo, J. Kuffner, K. Nishiwaki and S. Kagami, "Efficient prioritized inverse kinematic solutions for redundant manipulators," in Proceedings of IEEE/RSJ IROS, pp. 1905-1910, 2005.

[10] Y. Guan and K. Yokoi, "Reachable Space Generation of A Humanoid Robot Using The Monte Carlo Method," in Proceedings of IEEE/RSJ IROS, pp. 1984-1989, Oct. 2006.

[11] A. J. Caron, E. J. Kiel, M. Dayton, and S. C. Butler, "Comprehension of the referential intent of looking and pointing between 12 and 15 months," Journal of Cognition and Development, vol. 3(4), 2002, pp. $445-464$.

[12] S. Dunphy-Lelii, and H. M. Wellman, (2004) "Infants' understanding of occlusion of others' line-of-sight: Implications for an emerging theory of mind," European Journal of Developmental Psychology, vol 1(1), 2004, pp. 49-66.

[13] G. O. Deak, R. A. Flom, and A. D. Pick, "Effects of gesture and target on 12-and18-month-olds' joint visual attention to objects in front of or behind them," Developmental Psychology,.36(4), 2000, pp. 511-523.

[14] H. Moll, and M. Tomasello, "12-and18-month-old infants follow gaze to spaces behind barriers," Developmental Science, vol. 7(1), 2004, pp. F1-F9.

[15] D. L. Gardner, L. S. Mark, J. A. Ward, and H. Edkins, "How do task characteristics affect the transitions between seated and standing reaches?," Ecological Psychology, vol. 13, 2001, pp. 245-274.

[16] H. J. Choi, and L. S. Mark, "Scaling affordances for human reach actions," Human Movement Science, vol. 23, 2004, pp. 785-806.

[17] L. F. Marin-Urias, E. A. Sisbot, A. K. Pandey, R. Tadakuma, and R. Alami, "Towards shared attention through geometric reasoning for human robot interaction," IEEE-RAS International Conference on Humanoid Robots, pp. 331-336, Paris, France, 2009.

[18] M. Johnson, and Y. Demiris, "Perceptual Perspective Taking And Action Recognition," International Journal of Advanced Robotic Systems,Vol.2(4), Dec. 2005, pp 301-308.

[19] C. Breazeal, M. Berlin, A. Brooks, J. Gray, and A. L. Thomaz, "Using perspective taking to learn from ambiguous demonstrations," Robotics and Autonomous Systems, pp. 385-393, 2006.

[20] J. Gregory Trafton, Nicholas L. Cassimatis, Magdalena D. Bugajska, Derek P. Brock, Farilee Mintz, Alan C. Schultz, "Enabling effective human-robot interaction using perspective-taking in robots", IEEE Transactions on Systems, Man, and Cybernetics, 2005, 460-470.

[21] M. J. Richardson, K. L. Marsh, and R. M. Baron, "Judging and actualizing intrapersonal and interpersonal affordances," Journal of Experimental Psychology: Human Perception and Performance, vol. 33(4), Aug 2007, pp. 845-859.

[22] J. J. Gibson, The Ecological Approach to Visual Perception. Boston: Houghton Mifflin, 1979.

[23] D. A. Norman, "The Psychology of Everyday Things," New York: Basic Books, 1988.

[24] M. Lopes, F. S. Melo, and L. Montesano, "Affordance-based imitation learning in robots," IEEE/RSJ IROS, San Diego, USA, October 2007.

[25] R. Moratz, and T. Tenbrink, "Affordance-Based Human-Robot Interaction," Affordance-Based Robot Control, LNAI, vol. 4760, 2008, pp. 63-76.

[26] K. Kaneko, F. Kanehiro, S. Kajita, H. Hirukawa, T. Kawasaki, M. Hirata, K. Akachi, and T. Isozumi, "Humanoid robot hrp-2," IEEE ICRA, New Orleans, April 2004.

[27] S. Lemaignan, R. Ros, L. Mosenlechner†, R. Alami and M. Beetz, "ORO, a knowledge management module for cognitive architectures in robotics", submitted to IROS 2010.

[28] S. Cambon, R. Alami and F. Gravot, "A hybrid approach to intricate motion, manipulation and task planning." The International Journal of Robotics Research, 28(1):104-126, 2009.

[29] F. Zacharias, Ch. Borst and G. Hirzinger, "Bridging the Gap between Task Planning and Path Planning," IROS, pp.4490-4495, China, 2006.

[30] S. Alili, R. Alami, V. Montreuil, "A Task Planner for an Autonomous Social Robot," DARS, Tsukuba, 2008. 